that GSK-3 $\beta$ regulates the MPT (9). The MPT is poorly understood, and the precise molecular composition of the MPT needs to be defined (16). Advancement in cardioprotection and apoptosis will require a better understanding of the composition and regulation of the MPT and its role in apoptosis. Perhaps the most important issue is whether inhibition of GSK-3 $\beta$ is protective if it is initiated upon reperfusion. Inhibition of several kinases upon reperfusion has been reported to block protection afforded by insulin or other protective drugs administered upon reperfusion. Since these kinases phosphorylate GSK-3 $\beta$, it is plausible that GSK-3 $\beta$ is a target during reperfusion. Consistent with this, a recent study by Gross et al. (11) showed that addition of GSK-3 $\beta$ inhibitors 5 minutes before the start of reperfusion resulted in a significant reduction in infarct size.

Address correspondence to: Elizabeth Murphy, Laboratory of Signal Transduction, National Institute of Environmental Health Sciences, National Institutes of Health, 111
Alexander Drive, Research Triangle Park, North Carolina 27709, USA. Phone: (919) 541-3873; Fax: (919) 541-3385; E-mail: murphy1@niehs.nih.gov.

1. Murphy, E. 2004. Primary and secondary signaling pathways in early preconditioning that converge on the mitochondria to produce cardioprotection. Circ. Res. 94:7-17.

2. Hausenloy, D.J., and Yellon, D.M. 2004. New directions for protecting the heart against ischemiareperfusion injury: targeting the Reperfusion Injury Salvage Kinase (RISK) pathway. Cardiovasc. Res. 61:448-460.

3. Akao, M., Teshima, Y., and Marban, E. 2002. Antiapoptotic effect of nicorandil mediated by mitochondrial ATP-sensitive potassium channels in cultured cardiac myocytes. J. Am. Coll. Cardiol. 40:803-810.

4. Javadov, S.A., et al. 2003. Ischemic preconditioning inhibits opening of mitochondrial permeability transition pores in the reperfused rat heart. J. Physiol. 549:513-524.

5. Hausenloy, D.J., Maddock, H.L., Baxter, G.F., and Yellon, D.M. 2002. Inhibiting mitochondrial permeability transition pore opening: a new paradigm for myocardial preconditioning? Cardiovasc. Res. 55:534-543.

6. Korge, P., Honda, H.M., and Weiss, J.N. 2002. Protection of cardiac mitochondria by diazoxide and protein kinase C: implications for ischemic preconditioning. Proc. Natl. Acad. Sci. U. S. A. 99:3312-3317.

7. Baines, C.P., et al. 2003. Protein kinase C $\varepsilon$ interacts with and inhibits the mitochondrial transition pore in cardiac mitochondria. Circ. Res. 92:873-880.

8. Baines, C.P., et al. 2002. Mitochondrial PKC $\varepsilon$ and MAPK form signaling modules in the murine heart: enhanced mitochondrial PKCE-MAPK interactions and differential MAPK activation in PKCE-induced cardioprotection. Circ. Res. 90:390-397.

9. Juhaszova, M., et al. 2004. Glycogen synthase kinase$3 \beta$ mediates convergence of protection signaling to inhibit the mitochondrial permeability transition pore. J. Clin. Invest. 113:1535-1549. doi:10.1172/ JCI200419906.

10. Tong, H., Imahashi, K, Steenbergen, C., and Murphy, E. 2002. Phosphorylation of glycogen synthase kinase- $3 \beta$ during preconditioning through a phosphatidylinositol-3-kinase dependent pathway is cardioprotective. Circ. Res. 90:377-379.

11. Gross, E.R., Hsu, A.K., and Gross, G.J. 2004. Opioid-induced cardioprotection occurs via glycogen synthase kinase $\beta$ inhibition during reperfusion in intact rat hearts. Circ. Res. 94:960-966.

12. Cohen, P., and Frame, S. 2001. The renaissance of GSK-3. Nat. Rev. Mol. Cell Biol. 2:769-776.

13. Jope, R.S. 2003. Lithium and GSK-3: one inhibitor, two inhibitory actions, multiple outcomes. Trends Pharmacol. Sci. 24:441-443.

14. Hausenloy, D.J., Yellon, D.M., Mani-Babu, S., and Duchen, M.R. 2004. Preconditioning protects by inhibiting the mitochondrial permeability transition. Am. J. Physiol. doi:10.1152/1jpheart.00678.2003.

15. Inagaki, K., et al. 2003. Inhibition of $\delta$-protein kinase $\mathrm{C}$ protects against reperfusion injury of the ischemic heart in vivo. Circulation. 108:2304-2307.

16. Kakoszka, J.E., et al. 2004. The ADP/ATP translocator is not essential for the mitochondrial permeability transition pore. Nature. 427:461-465.

\title{
A fork in the road of cell differentiation in the kidney tubule
}

\author{
Qais Al-Awqati' and George J. Schwartz²
}

1Departments of Medicine and of Physiology and Cellular Biophysics, College of Physicians and Surgeons, Columbia University, New York, New York, USA.

${ }^{2}$ Departments of Pediatrics and of Medicine, University of Rochester, Rochester, New York, USA.

\begin{abstract}
The collecting ducts of the kidney are composed of intercalated cells (responsible for acid/base transport), principal cells (mediating salt and water absorption), and inner medullary cells, which mediate all three types of transport. Forkhead box (Fox) genes are a large family of transcription factors that are important in cell-type specification during organogenesis. In this issue, Blomqvist et al. find that mice lacking Foxi1 have no intercalated cells in the kidney (see the related article beginning on page 1560). The collecting ducts of the null mice contained primitive cells that expressed both intercalated cell and principal cell proteins, yet the acid/base transport function of the kidney was disrupted and the mice exhibited distal renal tubular acidosis. These findings suggest that Foxi1 plays a critical role in determining cell identity during collecting duct development.
\end{abstract}

The mechanism of generation of the 400 or more cell types of mammals from a single cell remains the principal problem of develop-

Conflict of interest: The authors have declared that no conflict of interest exists.

Citation for this article: J. Clin. Invest. 113:1528-1530 (2004). doi:10.1172/JCI200422029. mental cell biology. Classical embryologists discovered that progenitor cells first undergo specification before they become determined to form a differentiated cell type. Under certain conditions, specified cells can differentiate in a reversible manner, whereas determined cells autonomously differentiate even if placed in a different location in the embryo. The molec- ular mechanism of these changes in cell fate depends on signals received from the niche or "ecosystem" in which the cell is located, but ultimately it must involve the activation of a network of transcription factors that is specific for each tissue and cell type.

\section{Renal collecting duct}

Each nephron of the adult mammalian kidney is composed of 12 epithelial segments. The collecting duct has three segments, some of which have more than one cell type. These tubular segments begin to develop when the ureteric bud (an outgrowth of the wolffian duct) invades the metanephric mesenchyme. The tip of each ureteric bud induces the conversion of this mesenchyme into an epithelial structure that undergoes morphogenetic transformation to form the nephron from the glomerulus to the end of the distal tubule, while the ureteric bud 
forms the collecting duct. The adult collecting duct has several cell types, one of which - the intercalated cell - is specialized for the transport of $\mathrm{H}^{+}$and $\mathrm{HCO}_{3}{ }^{-}$. The other type - the principal cell-mediates salt and water transport. A third cell type, present only in the inner medullary collecting duct, performs salt, water, and acid transport. All of these cells derive from the ureteric bud, but the pathway of their differentiation remains obscure. The paper by Blomqvist et al. in this issue of the JCI is the first to provide insight into the molecular mechanism of cell-identity determination of at least the intercalated cell (1).

\section{Intercalated cells}

The intercalated cell exists in two functionally distinct subtypes in the cortical collecting duct: one secretes $\mathrm{HCO}_{3}{ }^{-}$, while the other absorbs it. Reabsorption of $\mathrm{HCO}_{3}{ }^{-}$ (i.e., secretion of protons) is accomplished in $\alpha$-intercalated cells by an apical $\mathrm{H}^{+}$-ATPase and a basolateral $\mathrm{Cl}^{-} / \mathrm{HCO}_{3}{ }^{-}$exchanger. Secretion of $\mathrm{HCO}_{3}{ }^{-}$by $\beta$-intercalated cells is mediated by the same kinds of transporters but located on the opposite cell membranes. We found that when animals were fed an acid diet, the $\beta$ form converted to the $\alpha$ phenotype (2). Others have shown, using a variety of methods, that there are more than two subtypes of intercalated cells, but we interpret these findings as showing a continuity of changes taking place between the two extremes of $\alpha$ and $\beta$. Recent studies based on single identified $\beta$-intercalated cells showed that these cells converted to an $\alpha$ phenotype in response to acidification of the basolateral media (3). Recently, we found in an intercalated cell line that this conversion represents an instance of terminal differentiation, with the $\beta$-intercalated cell representing a less differentiated (protoepithelial) type and the $\alpha$ phenotype being more differentiated (4).

\section{Foxi1}

Blomqvist et al. (1) find that mice lacking Foxi1, a forkhead transcription factor, have no intercalated cells as judged by electron microscopy, or by the absence of specific proteins of these cells. Foxi1 is expressed in the intercalated cell and in the inner ear. Could the transcription factor be a critical determinant of cell identity of these cells? The authors show a remarkable result: each cell of the collecting ducts of the mutant mice expresses both intercalated proteins (carbonic anhydrase II) and principal cell proteins (aquaporin 2). It would have been useful to find out whether the embryonic collecting duct also resembled the mutant ducts in their pattern of expression. Although studies using these excellent markers have not been performed, the entire collecting duct seems to be composed of a single cell type, staining weakly for aquaporin 2 (K. Madsen, personal communication).

\section{Development of the collecting duct}

Nephrogenesis proceeds in mice, rats, and rabbits for one week postnatally. Electron microscopic studies have shown that the cortical collecting duct of the newborn shows a single cell type, resembling a principal cell, with a single cilium, few organelles, smooth apical and basolateral surfaces, and large glycogen deposits (5). No intercalated cells can be demonstrated in the developing neonatal collecting duct, using physiological criteria (6). Minuth and colleagues found that the early collecting tubule contains a "scrambled" expression of principal and intercalated lectin-binding sites (7). But it remains to be shown definitively that development of the collecting tubule lineage progresses from a generic ureteric bud cell to a specified collecting tubule cell that finally terminally differentiates into principal, intercalated, and inner medullary collecting duct types. Incidentally, the latter cell contains both principal and intercalated cell proteins, which raises the question of whether these cells have been arrested in their developmental program at the specification stage.

\section{A kidney-ear axis?}

There is an interesting connection between renal anomalies and deafness. Deletion of ATP6B, of pendrin, and of $\mathrm{Kcc} 4$, the $\mathrm{K}-\mathrm{Cl}$ cotransporter present in intercalated cells, can cause deafness. One of the supporting cells of the sensory epithelium in the vestibular epithelium has many similarities to the intercalated cell, and it serves to control the acidic $\mathrm{pH}$ of the endolymph. Remarkably, deletion of Foxi1 depletes the epithelium of these cells, causing deafness (8).

\section{Forkhead genes and intercalated cell identity}

Forkhead genes (termed Fox, for forkhead box) encode a subset of the helix-turn-helix class of transcription factors (9). They are divided into 17 classes (A to $\mathrm{Q}$ ) and are involved in a wide range of cell-type specification during organogenesis in animals and humans. In Caenorhabditis elegans, a forkhead protein, pha-4 (an orthologue of
FoxA), specifies the identity of the pharynx and causes the expression of all pharyngeal genes (10). Remarkably, the order of appearance of the pharyngeal genes depends on the affinity of the promoters to the forkhead protein, providing an elegant mechanism that controls the timing of appearance of these genes.

Foxi1 controls the expression of pendrin and the band 3 anion exchangers (AE1), and probably also the ATP6B subunit of the vacuolar $\mathrm{H}^{+}$-ATPase, all characteristic proteins of the intercalated cells. But the absence of this factor resulted not only in the absence of these genes but also in the lack of this cell type. The intercalated cells contain a very large number of mitochondria, and their shape is quite characteristic because of the presence of vigorous apical endocytosis and exuberant microvilli. It seems unlikely that all of these characteristics are secondary events produced by expression of a few transport proteins. One possibility is that absence of the transport proteins leads to cell death during development. But since the expression of carbonic anhydrase and aquaporin 2 becomes scrambled in the mutant mice, it is likely that Foxi1 activates a whole program of expression of genes that control cell shape, organellar biogenesis, apical structure (microvilli and microplicae), and apical functions (endocytosis and exocytosis). Using microarray analysis it should be possible to identify the developmental program that specifies this cell type. But what is the developmental pathway by which Foxi1 determines the identity of the intercalated cell? Blomqvist et al. favor the idea that it has a simple and direct effect of cell determination similar to the proposed mechanism of pha-4 (1). However, it appears that Foxi1 is expressed in the kidneys at embryonic day 16 in a uniform pattern in the ureteric bud and collecting duct (ref. 11; note that this is our interpretation of Figure 2A of ref. 11, which differs from the opinion of the authors). This raises the question of whether Foxi1 plays a more complex role in cell specification in the collecting duct. For instance, its disappearance could also mediate principal cell differentiation. Forkhead genes often collaborate with other transcription factors to control the timing of differentiation, exerting negative and positive influences on these processes. For instance, Foxe1 collaborates with pax8 and TTF1 to control the differentiation of the thyroid cell. Foxe1 mutations result in thyroid agenesis (12), yet the gene is expressed in the thyroid 
before the differentiation of thyrocytes and is suppressed when thyroglobulin begins to be expressed (13). What seems clear is that the identification of Foxi1's role in intercalated cell differentiation has opened the door to an exciting new chapter in the development of the kidney.

Address correspondence to: Qais AlAwqati, Departments of Medicine and of Physiology and Cellular Biophysics, College of Physicians and Surgeons, Columbia University, 630 West 168th Street, New York, New York 10032, USA. Phone: (212) 305-3512; Fax: (212) 305-3475; E-mail: qa1@columbia.edu.

1. Blomqvist, S.R., et al. 2004. Distal renal tubular acidosis in mice that lack the forkhead transcrip- tion factor Foxi1. J. Clin. Invest. 113:1560-1570. doi:10.1172/JCI200420665.

2. Schwartz, G.J., Barasch, J., and Al-Awqati, Q. 1985. Plasticity of functional epithelial polarity. Nature. 318:368-371.

3. Schwartz, G.J., et al. 2002. Acid incubation reverses the polarity of intercalated cell transporters, an effect mediated by hensin. J. Clin. Invest. 109:89-99. doi:10.1172/JCI200213292.

4. Vijayakumar, S., Takito, J., Hikita, C., and Al-Awqati, Q. 1999. Hensin remodels the apical cytoskeleton and induces columnarization of intercalated epithelial cells: processes that resemble terminal differentiation. J. Cell Biol. 144:1057-1067.

5. Evan, A.P., Satlin, L.M., Gattone, V.H., 2nd, Connors, B., and Schwartz, G.J. 1991. Postnatal maturation of rabbit renal collecting duct. II. Morphological observations. Am. J. Physiol. 261:F91-F107.

6. Satlin, L.M., and Schwartz, G.J. 1987. Postnatal maturation of rabbit renal collecting duct: intercalated cell function. Am. J. Physiol. 253:F622-F635.

7. Aigner, J., Kloth, S., Jennings, M.L., and Minuth, W.W. 1995. Transitional differentiation patterns of principal and intercalated cells during renal collect- ing duct development. Epithelial Cell Biol. 4:121-130. 8. Hulander, M., et al. 2003. Lack of pendrin expression leads to deafness and expansion of the endolymphatic compartment in inner ears of Foxi1 null mutant mice. Development. 130:2013-2025.

9. Lehmann, O.J., Sowden, J.C., Carlsson, P., Jordan, T., and Bhattacharya, S.S. 2003. Fox's in development and disease. Trends Genet. 19:339-344.

10. Gaudet, J., and Mango, S.E. 2002. Regulation of organogenesis by the Caenorhabditis elegans FoxA protein PHA-4. Science. 295:821-825.

11. Overdier, D.G., Ye, H., Peterson, R.S., Clevidence, D.E., and Costa, R.H. 1997. The winged helix transcriptional activator HFH-3 is expressed in the distal tubules of embryonic and adult mouse kidney. J. Biol. Chem. 272:13725-13730.

12. Clifton-Bligh, R.J., et al. 1998. Mutation of the gene encoding human TTF-2 associated with thyroid agenesis, cleft palate and choanal atresia. Nat. Genet. 19:399-401.

13. Zannini, M., et al. 1997. TTF-2, a new forkhead protein, shows a temporal expression in the developing thyroid which is consistent with a role in controlling the onset of differentiation. EMBOJ. 16:3185-3197.

\title{
The case of visceral fat: argument for the defense
}

\section{Samuel Klein}

Center for Human Nutrition and Division of Geriatrics and Nutritional Sciences, Washington University School of Medicine, St. Louis, Missouri, USA.

\begin{abstract}
Increased plasma fatty acid concentrations may be responsible for many of the metabolic abnormalities associated with abdominal obesity. Excessive visceral fat is associated with insulin resistance and other metabolic risk factors for coronary heart disease. A study reported in this issue of the JCI evaluates the relative contribution of fatty acids released during lipolysis of visceral adipose tissue triglycerides to portal and systemic fatty acid flux in human subjects (see the related article beginning on page 1582).
\end{abstract}

The relationship between excess abdominal fat mass and insulin resistance was recognized a half century ago, when Jean Vague, a French physician, reported an association between a "masculine" or "android" obesity phenotype and diabetes (1). Subsequently, many large epidemiological and smaller physiological studies have confirmed the relationship between abdominal obesity and insulin resistance, diabetes, and other metabolic risk factors for coronary heart disease (2-5). In fact, excess abdominal fat is even associated with impaired insulin-mediated glucose uptake in lean adults (6).

Abdominal fat is composed of several distinct anatomic depots: subcutaneous fat, which can be divided into anterior and posterior or superficial and deep layers, and intraabdominal fat, which can be divided

Conflict of interest: The author has declared that no conflict of interest exists.

Citation for this article: J. Clin. Invest. 113:1530-1532 (2004). doi:10.1172/JCI200422028. into intraperitoneal and retroperitoneal sites. Intraperitoneal fat, also known as visceral fat, is composed of mesenteric and omental fat masses. Although the absolute amount of each of these depots is much larger in upper-body obese than in lean persons, the relative amount of abdominal fat with respect to total body fat mass is often similar in both groups. For example, visceral fat constitutes about $10 \%$ of total body fat mass in lean and obese men (7).

The close relationship between abdominal fat (i.e., total, subcutaneous, and/or visceral fat) and metabolic disease has stimulated a clinical interest in identifying high-risk patients. Waist circumference is often used as a surrogate marker of abdominal fat because it correlates closely with total abdominal fat mass measured by computed tomography (8) and it is not practical to directly measure abdominal fat mass in a clinical setting. Based on data from epidemiological studies, the Expert Panel on the Identification, Evaluation, and Treatment of Overweight and
Obesity in Adults, convened by the NIH, proposed that men with a waist circumference greater than $102 \mathrm{~cm}$ (40 in.) and women with a waist circumference greater than $88 \mathrm{~cm}$ (35 in.) are at increased risk for metabolic diseases (9).

\section{Fatty acid metabolism and insulin resistance}

The association between abdominal fat and insulin resistance does not prove causality, and it is possible that environmental, biological, or inherited factors that induce insulin resistance also cause abdominal fat accumulation (10). Nonetheless, it has been proposed that alterations in fatty acid metabolism associated with abdominal obesity are responsible for impaired insulin action because excessive circulating FFAs inhibit the ability of insulin to stimulate muscle glucose uptake and to suppress hepatic glucose production (11). The notion of a link between abdominal fat, FFA metabolism, and insulin resistance is supported by the observation that basal wholebody FFA flux rates are greater in upperbody obese than in lower-body obese and lean subjects $(12,13)$ and that diet-induced weight loss decreases whole-body FFA flux and improves insulin sensitivity (14). It has been hypothesized that excess visceral fat is more harmful than excess subcutaneous fat, because lipolysis of visceral adipose tis- 